\section{Homology between IgE-binding factor and retrovirus genes}

THE recent report by Toh et al. ${ }^{1}$ of sequence homology between segments of a cloned gene encoding rodent IgEbinding factor (IgE-BF) ${ }^{2}$ and a gene encoding Syrian hamster intracisternal Aparticle (IAP) ${ }^{3}$ is an intriguing observation. However, we must express reservations about Toh et al.'s interpretations of the observed homology.

First, the authors claim that because the $\mathrm{N}$-terminal third of the IgE-BF coding sequence shows no homology with their particular IAP gene, the IgE-BF gene must be "a hybrid gene which evolved ... by integrating genes of viral origin". No evidence is presented by either Toh et al. or Martens et al. ${ }^{2}$ in support of the authors' assertions that the cloned IgE-BF gene is a hybrid gene or that it even contains a portion of a non-IAP gene at all. Neither report presents any genomic blot data to indicate whether homologous genes exist in the mouse or rat genomes. Here, the conclusions of Toh et al. are considerably weakened by the fact that their sequence comparison crosses species lines; they really have no justification for making any statement regarding the nature of IgE-BF sequences which do not show homology to their hamster IAP sequence. Thus, their assertion that the $\mathrm{N}$-terminal third of the $\mathrm{IgE}-\mathrm{BF}$ amino-acid sequence is unique to IgE-BF is speculative and not supported by their data.

The IgE-BF clone was derived by Martens et al. from a rat-mouse T-cell hybridoma. Toh et al. do not mention the possibility that the IgE-BF clone could represent a hybridoma-related recombination event, as opposed to an evolutionarily significant insertion of a retroviral-related sequence into "a cellular DNA region proximal (sic) to a primordial IgE-BF gene". Observations suggesting such an alternative explanation exist in the literature ${ }^{4-6}$. These data all demonstrate that in the mouse genome, IAP genes are mobile elements which can affect the expression of unrelated genes. Toh et al. overlooked the possibility that the structure of the cloned IgE-BF gene could have arisen by insertion (in the hybridoma) of an IAP sequence distal to the IgE-BF coding sequence, followed by transcription of this region and splicing of the " 'IgE-BF', gag and pol" sequences. In view of this possibility, it is important to stress that the IgE-BF clone is a complementary DNA, rather than a genomic clone; Toh et al. make inferences of considerable import about the structure and evolution of a putative genomic gene from sequence comparisons involving only a cDNA clone.

Taken together, the arguments outlined above also cast doubt on the statement of

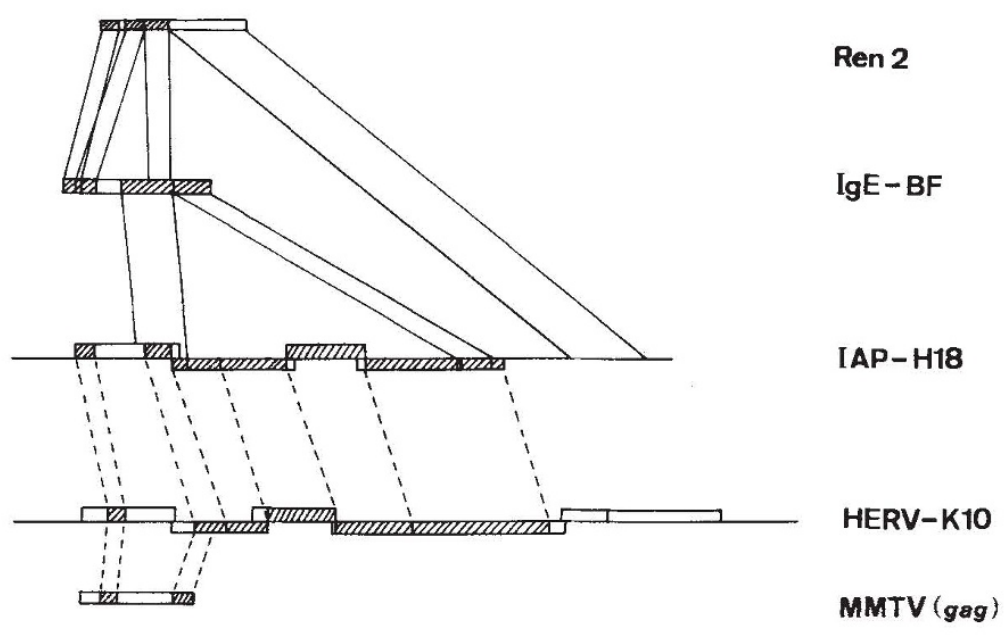

Fig. 1 Profiles of sequence homology of IgE-BF cDNA to mouse (Ren 2) and hamster (H-18) IAPs, human endogenous retrovirus (HERV-K10) and MMTV gag. Homologous regions are indicated by slanted lines. Regions where homologies exist at the DNA level and at the protein level are connected by vertical straight lines and dotted lines, respectively.

Toh et al. that a particular proteolytic cleavage of the precursor IgE-BF is more likely than another to generate the $11 \mathrm{~K}$ (relative molecular mass 11,000) IgE-BF from the $60 \mathrm{~K}$ species.

Thus, we believe that while the observations of Toh et al. are of interest, conclusions about their possible significance are speculative, and certainly premature in the absence of any experimental data examining the relationship of IgE-BF to IAP. A preliminary report of the homology of IgE-BF to mouse IAP genes appeared earlier ${ }^{7}$, but was not cited by Toh et al.

\section{KEVIN W. MOORE}

Christine L. MARTENS

Department of Immunology,

DNAX Research Institute of

Molecular and Cellular Biology,

Palo Alto,

California 94304, USA

1. Toh, H., Ono, M. \& Miyata, T. Nature 318, 388-389 (1985). 2. Martens, C. L. et al. Proc. natn. Acad. Sci. U.S.A. 82, 2460-2464 (1985)

3. Ono, M., Toh, H., Miyata, T. \& Awaya, T. J. Virol. 55, 387-394 (1985).

4. Hawley, R. G., Shulman, M. J., Murialdo, H., Gibson, D. M. \& Hozumi, N. Proc. natn. Acad. Sci. U.S.A. 79, 74257429 (1982).

5. Kuff, E. L. et al. Proc. natn. Acad, Sci. U.S.A. 80, 1992-1996 (1983).

6. Kuff, E. L. et al. Nature 302, 547-548 (1985).

7. Martens, C. et al. in Immune Regulation (eds Feldmann, M. \& Mitchison, N. A.) 121-130 (Humana, Clifton, New Jersey, 1985).

MIYATA ET AL. REPLY-We have shown previously $^{1}$ that the IgE-BF cDNA from a rat-mouse T-cell hybridoma ${ }^{2}$ exhibits a strong sequence homology to the Syrian hamster IAP ${ }^{3}$ at the nucleotide level in the $3^{\prime}$ two-thirds of the protein-coding region, whereas the remaining 5 one-third shows no obvious homology. This non- homologous region of the IgE-BF cDNA, however, shows sequence homology to a mouse IAP (Ren 2$)^{4}$ at the DNA level, but not to a distantly related human endogenous retrovirus, HERV-K10 (M.O. et al., in preparation) or to a mouse mammary tumour virus (MMTV) ${ }^{5}$ gag region, both of which share obvious homology with the hamster IAP at the protein level (Fig. 1). Moore et al. ${ }^{6}$ recently demonstrated sequence homology of the IgE-BF cDNA to mouse IAPs, represented by MIA14, over its entire regions. These results suggest that the nucleotide sequence corresponding to the $5^{\prime}$ third of the IgE-BF coding region is unique to mouse IAPs or their close relatives. This unique region might have been derived very recently from a distantly related retrovirus or an endogenous retrovirus by a recombination mechanism, or from a primordial IgE-BF gene of cellular origin, as suggested previously ${ }^{1}$.

TAKASHI MIY
HIROYUKI TO
Department of Biology,
Faculty of Science,
Kyushu University,
Fukuoka 812, Japan
$\quad$ MASAO ONO
Department of Molecular Biology,
School of Medicine,
Kitasato University,
Kanagawa 228, Japan

1. Toh, H., Ono, M. \& Miyata, T. Nature 318, 388-389 (1985). 2. Martens, C. L. et al. Proc. natn. Acad. Sci. U.S.A. 82, 2460-2464 (1985).

3. Ono, M., Toh, H., Miyata, T. \& Awaya, T. J. Virol. 55, 387-394 (1985).

4. Burt, D. W., Reith, A. D. \& Brammar, W. J. Nucleic Acids Res. 12, 8579-8593 (1984).

5. Fasel, N. et al. Nucleic Acids Res. 11, 6943-6955 (1983)

6. Moore, K. W. et al. J. Immun. 136, 4283-4290 (1986). 